\title{
TBK1 mutations in Italian patients with amyotrophic lateral sclerosis: genetic and functional characterisation
}

\author{
Laura Pozzi, ${ }^{1}$ Fabiola Valenza, ${ }^{2}$ Lorena Mosca, ${ }^{3}$ Andrea Dal Mas, ${ }^{2}$ Teuta Domi, ${ }^{1}$ \\ Alessandro Romano, ${ }^{1}$ Claudia Tarlarini, ${ }^{3}$ Yuri Matteo Falzone, ${ }^{1,4}$ Lucio Tremolizzo, ${ }^{5}$ \\ Gianni Sorarù, ${ }^{6}$ Federica Cerri, ${ }^{1,4}$ Pilar M Ferraro, ${ }^{7}$ Silvia Basaia, ${ }^{7}$ Federica Agosta, ${ }^{7}$ \\ Raffaella Fazio, ${ }^{4}$ Mauro Comola, ${ }^{4}$ Giancarlo Comi, ${ }^{4,8}$ Maurizio Ferrari, 8,9 \\ Angelo Quattrini, ${ }^{1}$ Christian Lunetta, ${ }^{10}$ Silvana Penco, ${ }^{3}$ Dario Bonanomi, ${ }^{2}$ \\ Paola Carrera, ${ }^{9}$ Nilo Riva ${ }^{1,4}$
}

- Additional material is published online only. To view please visit the journal online (http://dx.doi.org/10.1136/ jnnp-2017-316174)

For numbered affiliations see end of article.

Correspondence to Dr Nilo Riva, Department of Neurology, INSPE and Division of Neuroscience, San Raffaele Scientific Institute, via Olgettina 48, 20132 Milan, Italy; riva. nilo@hsr.it

Received 31 March 2017 Revised 31 May 2017 Accepted 23 June 2017 Published Online First 19 August 2017

\section{CrossMark}

To cite: Pozzi L, Valenza F, Mosca L, et al. J Neurol Neurosurg Psychiatry 2017:88:869-875.

\section{ABSTRACT}

Background TANK-binding kinase 1 (TBK1) gene has been recently identified as a causative gene of amyotrophic lateral sclerosis (ALS).

Methods We sequenced the TBK1 gene in a cohort of 154 Italian patients with ALS with unclear genetic aetiology. We subsequently assessed the pathogenic potential of novel identified TBK 1 variants using functional in vitro studies: expression, targeting and activity were evaluated in patient-derived fibroblasts and in cells transfected with mutated-TBK1 plasmids.

Results We identified novel genomic TBK1 variants including two loss-of-function (LoF) (p.Leu59Phefs*16 and c.358+5G>A), two missense (p.Asp118Asn and p.lle397Thr) and one intronic variant (c.1644-5_16442delAATA), in addition to two previously reported pathogenetic missense variants (p.Lys291Glu and p.Arg357GIn). Functional studies in patientderived fibroblasts revealed that the c.358+5G $>$ A causes aberrant pre-mRNA processing leading TBK1 haploinsufficiency. Biochemical studies in cellular models showed that the truncating variant p.Leu59Phefs * 16 abolishes TBK1 protein expression, whereas the p.Asp118Asn variant severely impairs TBK1 phosphorylation activity. Conversely, the p.lle397Thr variant displayed enhanced phosphorylation activity, whose biological relevance is not clear.

Conclusion The observed frequency of TBK1 LoF variants was $1.3 \%(2 / 154)$, increasing up to $3.2 \%$ $(5 / 154)$ by taking into account also the functional missense variants that we were able to classify as potentially pathogenic, supporting the relevance of TBK1 in the Italian population with ALS.

\section{INTRODUCTION}

Amyotrophic lateral sclerosis (ALS) is a severe neurodegenerative motor neuron disease characterised by progressive loss of upper and lower motor neurons leading to death within $2-5$ years after diagnosis. ${ }^{1}$ Cognitive dysfunction occurs in $20 \%-50 \%$ of cases, whereas $5 \%-15 \%$ of patients develop overt frontotemporal dementia (FTD). ${ }^{23}$ Most ALS cases are apparently sporadic
(sALS), while in approximately $10 \%$ of patients, a positive family history can be identified (fALS). ${ }^{145}$ Although more than 50 potential ALS-related genes have been reported so far, a genetic aetiology may be determined in a minority of patients with sALS and in about two-thirds of fALS cases, with pathogenic variants in the SOD1, FUS and TARDBP, and repeat expansion in C9orf72 being the most common. ${ }^{46}$

Recent exome sequencing studies revealed TANKbinding kinase 1 (TBK1, MIM no 604834) as a novel causative gene of both $\mathrm{ALS}^{78}$ and FTD, ${ }^{9}$ suggesting haploinsufficiency as a disease mechanism. TBK1 encodes a multifunctional kinase protein that phosphorylates a wide range of substrates and exerts control over several cellular key processes, including innate immune response, inflammation, autophagy and cell proliferation. ${ }^{10}{ }^{11}$ Notably, TBK1 interacts with optineurin (OPTN), ${ }^{12}$ valosin-containing protein and sequestosome-1 (SQSTM/P62), ${ }^{13}$ which have previously been reported as causative ALS genes. ${ }^{11}$

In this study, we have investigated the genetic role of TBK1 variants in a cohort of Italian patients with ALS and addressed their pathogenic potential using functional in vitro studies.

\section{MATERIALS AND METHODS \\ Patients}

The TBK1 gene was sequenced in a cohort of 154 unrelated Italian patients with ALS. Blood samples were obtained for diagnostic purposes, after informed consent, and stored in our tissue bank; the study protocol was approved by the local ethic committee. ALS diagnosis was made according to El Escorial revised criteria. ${ }^{14}$ Patients with definite, probable, probable laboratory supported or possible ALS were included in the study. Although no strict inclusion or exclusion criteria were adopted, patients with earlier disease onset, fALS or cognitive impairment were prioritised for TBK1 sequencing by referring clinicians. Thirty patients $(19.5 \%)$ had fALS, ${ }^{15}$ whereas 16 patients $(10.4 \%)$ had a positive family history for FTD and 31 (20.1\%) had cognitive impairment or unclassified 
dementia. The presence of pathogenic variants in known ALS genes was excluded by Sanger sequencing (SOD1, TARDBP and FUS/TLS) or repeat-primed PCR (C9orf72). The mean onset age of our cohort was $54.9 \pm 15.3$ years (range $20-82$ years), that is slightly younger compared with previous population-based studies. $^{16}$ Eighty-seven patients $(56.5 \%)$ were males and 67 (43.5\%) were females. Hundred and sixteen $(75.3 \%)$ had spinalonset ALS and 38 (24.7\%) had bulbar-onset ALS. The mean ALS Functional Rating Scale-revised (ALSFRS-R) Score at the first visit was 31.4 \pm 10.4 (range 4-47). All patients were of Caucasian ethnicity, except one who was of Hispanic origin.

Finally, we performed a PubMed literature review of all previously reported patients harbouring TBK1 variants and extracted, when available, their clinical and demographic characteristics.

\section{Genetic analysis}

For all patients, TBK1 whole coding region and exon junctions were analysed with a Sanger protocol, using the Big Dye Terminator V.1.1 Cycle Sequencing Kit (Applied Biosystems) (online supplementary table 1 for primers). Called sequences were aligned to the TBK1 reference sequence (National Center for Biotechnology Information (NCBI) Entrez gene ID 29110; NM_013254.3) with the Sequencer V.5.0 software (gene codes). Gene variants were evaluated by their absence or frequency in the public NCBI genome database, single-nucleotide polymorphism database (dbSNP), ExAc (Exome Aggregation Consortium) and EVS (Exome Variant Server). The Human Gene Mutation Database (HGMD) Professional 2016.4, the ALSoD (Amyotrophic Lateral Sclerosis Online Database) and PubMed have been interrogated to check for previously reported variants. The SIFT, PolyPhen-2 and MutationTaster in silico softwares were used to assess the functional effect of missense variants. Human Splicing Finder, NetGene2, neural network site, MaxEntScan and Gene Splicer were used to evaluate the potential effects on gene splicing. Crystal structure-based analysis was performed in order to asses the impact of novel TBK1 missense variants on protein thermodynamic stability $(\Delta \Delta G)$ and electrostatic surface potentials. ${ }^{1718}$

\section{Transcript analysis and functional in vitro studies}

Total RNA was extracted from patients' peripheral blood samples, and transcript analysis was performed by RT-PCR to detect potential splicing defects of four TBK1 variants (online supplementary table 2 for primers). TBK $1 \mathrm{mRNA}$ and protein expression levels were quantified in patient-derived fibroblasts harbouring the c. $358+5 \mathrm{G}>\mathrm{A}$ variant, which were available for testing, by quantitative real-time PCR (qRT-PCR) and western blot. Expression, targeting and activity of the novel frameshift and missense TBK1 variants were studied in AD293 human embryonic kidney (HEK) cells and mouse motor neuron-like NSC-34 cells transfected with TBK1 plasmids containing the relevant mutations. Kinase activity of these variants was assessed by measuring the levels of phosphorylated interferon regulatory factor 3 (IRF3), a known substrate, while association with the canonical interactor OPTN was investigated with pull-down assays in transfected AD293 cells.

Extensive technical description is reported in online supplementary information.

\section{RESULTS}

Identification of the novel TBK1 variants

Overall, we identified seven TBK1 variants in our cohort of unrelated Italian index patients (table 1). The p.Leu59Phefs*16 and

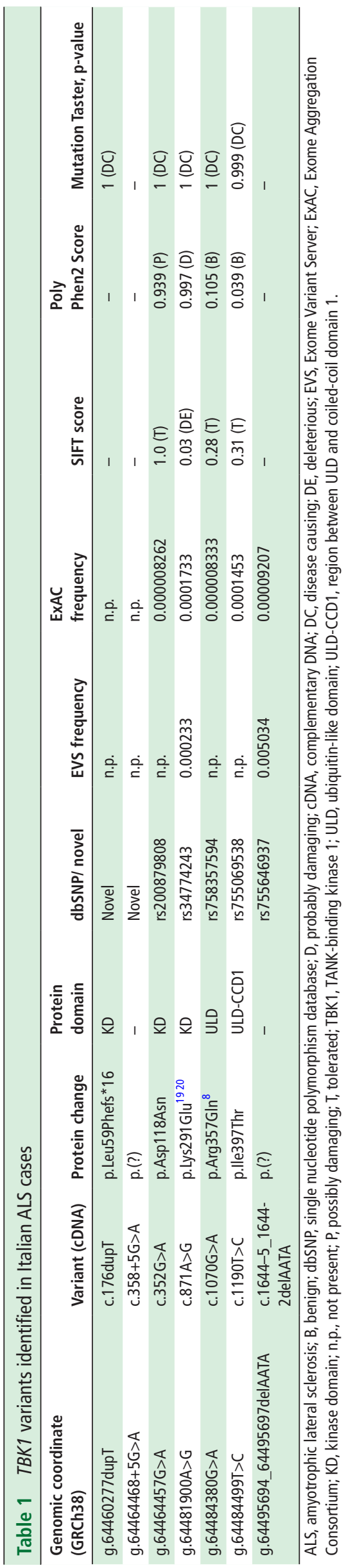


the c. $358+5 \mathrm{G}>\mathrm{A}$ are absent from all public genomic databases, including dbSNP. The missense p.Lys291Glu and p.Arg357Gln, located in the kinase domain (KD) and ubiquitin-like domain (ULD), respectively, have been previously reported as displaying an impairment in TBK1 catalytic activity ${ }^{819} 20$ and thus regarded as functional missense mutations, ${ }^{20}$ potentially pathogenic. The missense p.Asp118Asn and p.Ile397Thr, as well as the intronic variant c.1644-5_1644-2delAATA are reported in the general population as variants at very low frequency (table 1). None of them has been previously described in association to a clinical phenotype. The Exac and EVS frequencies for the P.Lys291Glu, c.1644_5_1644-2delAATA and p.Ile397Thr are greater than expected for the disorder (table 1), thus lowering their probability to be pathogenic.

The p.Leu59Phefs* 16 frameshift results in a putative truncated protein of 73 amino acids. Likewise, as predicted by splice-site in silico softwares, the intronic variant c. $358+5 \mathrm{G}>\mathrm{A}$ is expected to completely abolish the recognition of the donor splice site, five base pairs downstream exon 4 , leading to a putative aberrant protein product (online supplementary table 3 ). Thus, both mutations are predicted to be deleterious.

Conversely, in silico prediction analysis indicates that the intronic variant c.1644-5_1644-2delAATA does not affect the natural exon 15 acceptor site (online supplementary table 3 ). Moreover, this variant was also identified in the unaffected patient's mother whose DNA was available for testing, not supporting its pathogenic role.

The missense variant p.Asp118Asn, located in the KD, is predicted as deleterious by PolyPhen-2 and Mutation Taster. Moreover, structure-based analysis confirmed that the presence of 118Asn determines significant conformational and electrostatic surface potential changes in the TBK1 catalytic domain, reducing protein global stability $(\Delta \Delta \mathrm{G}: 3.98 \pm 0.27 \mathrm{kcal} /$ mol) (online supplementary figure $1 \mathrm{~A}, \mathrm{~B})$. In contrast, the p.Ile397Thr variant, located in the linker region between the ULD and the coiled-coil domain 1 (CCD1), is predicted to be tolerated and benign by SIFT and PolyPhen-2, but predicted to be disease causing by Mutation Taster (table 1). Protein structure analysis showed that the mutant 397Thr has little effects on
TBK1 structure and global stability $(\Delta \Delta \mathrm{G}: 1.51 \pm 0.18 \mathrm{kcal} / \mathrm{mol})$, with no changes in protein electrostatic surface potential (see online supplementary figure 1C,D). The p.Ile397Thr was absent in the patient's unaffected sister, whose DNA was available for testing. Finally, since at the genomic level the coding sequences for p.Asp118Asn and p.Ile397Thr are located in the proximity of donor and acceptor splice sites, respectively (online supplementary table 3), an in silico splicing prediction was performed. This evaluation predicted a potential weak impact of the two variants, not supporting a relevant alteration of the splicing process.

All the identified TBK1 variants were found in apparently patients with sALS, whose demographic and clinical characteristics are shown in table 2, while a summary of the main features of patients harbouring TBK1 gene variants reported so far is provided in table 3 (online supplementary table 4 for details).

\section{Effect of variants on $T B K 1$ transcript: the mutant c.358+5A allele disrupts processing of TBK1 transcript}

In order to assess the potential consequences on splicing predicted in silico (online supplementary table 3), we performed a transcript analysis on patient's peripheral blood samples. However, no aberrant transcripts were identified by RT-PCR and cDNA sequencing.

We were able to further investigated the intronic c. $358+5 \mathrm{G}>\mathrm{A}$ variant, since in silico analysis predicted inactivation of the canonical donor splice site (online supplementary table 3) could lead to exon 4 skipping, insertion of a premature termination codon (PTC) and degradation of the aberrant transcript through non-sense mediated decay (NMD) (figure 1A). In agreement with this hypothesis, quantitative RT-PCR analysis of TBK1 transcripts from blood and patient-derived primary fibroblasts showed a 50\% reduction in total mRNA levels (figure 1B). Immunoblot analysis of patient's fibroblasts confirmed a corresponding decrease in protein levels (figure 1D,E). Inhibition of NMD with cycloheximide restored normal levels of total TBK1 transcripts (figure 1B) and allowed detection of the aberrant isoform lacking exon 4 deriving from the mutated allele (figure 1C). Altogether, these results demonstrate that the

Table 2 Demographic and clinical characteristics of patients with TBK1 variants

\begin{tabular}{|c|c|c|c|c|c|c|c|c|}
\hline Variant & Sex & $\begin{array}{l}\text { Age of onset } \\
\text { (years) }\end{array}$ & Family history & $\begin{array}{l}\text { Disease duration } \\
\text { (months)* }\end{array}$ & $\begin{array}{l}\text { ALSFRS-R } \\
\text { progression } \\
\text { rate } \dagger\end{array}$ & Site of onset & Phenotype $^{16}$ & $\begin{array}{l}\text { Cognitive } \\
\text { impairment }^{25}\end{array}$ \\
\hline p.Leu59Phefs*16 & $\mathrm{F}$ & 36 & SALS & $52 \S$ & 0.92 & Spinal & Classic & No \\
\hline c. $358+5 G>A \ddagger$ & M & 62 & SALS & 17 & 0.7 & Spinal & PLMN & No \\
\hline p.Asp118Asnף & M & 81 & SALS & $35 \dagger$ & 0.6 & Spinal & PLMN & Yes (ALS-ECI) \\
\hline p.Lys291Gluq & M & 74 & SALS / $\Delta$ & 33 & 0.57 & Spinal & Flail arm** & Yes (ALS-NECI) \\
\hline p.Arg357GInף & $\mathrm{F}$ & 36 & SALS & 20 & 1.2 & Spinal & Classic & No \\
\hline p.lle397Thrł‡ & M & 65 & $S A L S / \varphi$ & 60 & 0.45 & Spinal & Pyramidal & Yes (ALS-ECI) \\
\hline $\begin{array}{l}\text { c.1644-5_1644- } \\
\text { 2delAATA } \ddagger\end{array}$ & M & 43 & SALS & 26 & 2.9 & Spinal & Pyramidal & No \\
\hline
\end{tabular}

*Disease duration: time from disease onset to latest visit or death.

tProgression rate $=(48-A L S F R S-R$ score $) /$ disease duration.

łLoss of function.

§Death.

ףPotentially pathogenic functional missense mutation.

**Hispanic ethnicity.

††Biological and pathogenetic relevance not clear by in vitro functional studies.

$¥ \neq$ Not demonstrated as pathogenic.

ALSFRS-R, ALS Functional Rating Scale-revised; ALS-ECl, ALS with executive cognitive impairment; ALS-NECI, ALS with non-executive cognitive impairment; PLMN, pure

lower motor neuron disease; sALS, sporadic amyotrophic lateral sclerosis; TBK1, TANK-binding kinase 1.

$\Delta:$ a brother and a sister deceased approximately at the age of 75 years reported to be affected by unclassified dementia. $\Phi$ : mother deceased at the age of 90 years reported to be affected by unclassified dementia. 
Table 3 Summary data of review of the literature of patients with TBK1 variants

\begin{tabular}{|c|c|c|c|c|c|c|c|c|}
\hline & \multirow{2}{*}{$\begin{array}{l}\text { No of } \\
\text { patients } \\
\text { (variants) }\end{array}$} & \multicolumn{3}{|c|}{ Clinical presentation* } & \multirow[t]{2}{*}{$\mathrm{M} / \mathrm{F}^{*}$} & \multicolumn{3}{|c|}{$\begin{array}{l}\text { Mean age at onset } \\
\pm S D \text { (years)* }\end{array}$} \\
\hline & & $\begin{array}{l}\text { ALS } \\
\text { (fALS) }\end{array}$ & $\begin{array}{l}\text { ALS- } \\
\text { FTD }\end{array}$ & FTD & & $\mathrm{M}+\mathrm{F}$ & M & $\mathrm{F}$ \\
\hline All & $\begin{array}{l}110 \\
(80)\end{array}$ & $\begin{array}{l}57 \\
\text { (17) }\end{array}$ & 16 & 19 & $45 / 39$ & $\begin{array}{l}60.4 \\
\pm 10.9\end{array}$ & $\begin{array}{l}58.1 \\
\pm 11.3\end{array}$ & $\begin{array}{l}62.0 \\
\pm 10\end{array}$ \\
\hline LoF & $\begin{array}{l}59 \\
(32)\end{array}$ & $\begin{array}{l}30 \\
(13)\end{array}$ & 10 & 12 & $30 / 24$ & $\begin{array}{l}62.5 \\
\pm 9.7\end{array}$ & $\begin{array}{l}61.0 \\
\pm 9.6\end{array}$ & $\begin{array}{l}64.3 \\
\pm 9.7\end{array}$ \\
\hline
\end{tabular}

${ }^{*}$ For some patients reported data are not complete. Extensive data are presented in online supplementary table 4 .

ALS, amyotrophic lateral sclerosis; F, female; fALS, familial ALS; FTD, frontotemporal dementia; LoF, loss of function; M, male; TBK1, TANK-binding kinase 1.

c. $358+5 \mathrm{G}>$ A genomic variant causes pre-mRNA misprocessing leading to TBK1 haploinsufficiency.

\section{Functional characterisation of TBK1 variants}

In order to assess the functional consequence of TBK1 variants, we introduced the p.Leu59Phefs*16 frameshift, the p.Asp118Asn and p.Ile397Thr missense variants in motor neuron-like NSC34 and HEK293T cell lines and studied their expression, targeting and activity. The p.Arg357Gln TBK1 missense, previously demonstrated as a potentially pathogenic functional missense mutation, was used as an experimental control. ${ }^{8}$

The p.Leu59Phefs*16 mutation leads to a putative truncated protein of 73 amino acids $(8.5 \mathrm{kDa}$ expected size) that was undetectable by immunofluorescence and western blotting (null allele) (figure 2A, B), whereas the p.Asp118Asn, p.Ile397Thr and p.Arg357Gln variants displayed a subcellular localisation virtually identical to wild-type TBK1 when expressed in motor neuron-like NSC34 cells (figure 2A). However, steady-state protein levels assessed by western blot were invariably lower for the p.Arg357Gln variant ( $\sim 60 \%$ reduction) and approximately $10 \%$ higher for the p.Ile397Thr variant, relative to wild-type TBK1, suggesting an effect on protein turnover. On the contrary, the levels of the p.Asp118Asn TBK1 were comparable to those of the wild-type protein (figure 2B, top panels).

To determine whether these missense variants might interfere with the kinase activity of TBK1, we quantified the phosphorylation of its target IRF3 at Ser-396. TBK1 harbouring the p.Asp118Asn variant in the KD yields reduced levels of phospho-IRF3 (p-IRF3) relative to wild-type TBK1. Therefore, the p.Asp118Asn can be regarded as a functional missense mutation, potentially pathogenic. In contrast, p-IRF3 levels were elevated in cells expressing the p.Ile397Thr variant, which is located in the linker region between the ULD and CCD1 domains (figure 2B, bottom panels). Interestingly, p-IRF3 levels were approximately $50 \%$ lower in cells expressing the p.Arg357Gln variant, located in the ULD, consistent with previous reports ${ }^{8}$; however, this decrease was related to a substantial reduction in the steady-state levels of this variant (figure 2B, top panels).

We finally examined the ability of TBK1 variants to interact with the autophagy receptor OPTN. Pull-down assays with tagged constructs revealed that the three TBK1 missense mutants (p.Asp118Asn, p.Ile397Thr and p.Arg357Gln) were competent for association with OPTN, whereas the p.Leu59Phefs"16, as expected, did not bind OPTN (figure 2C).

\section{DISCUSSION}

In order to understand the relevance of TBK1 as a disease gene in the Italian ALS population, we analysed a cohort of 154 unrelated patients with ALS by sequencing the whole coding region and exon junctions. We identified two loss of function (LoF) variants, the frameshift p.Leu59Phefs"16 and the intronic c. $358+5 \mathrm{G}>\mathrm{A}$, never reported before. In addition, we found two missense, p.Arg357Gln and p.Lys291Glu, previously described in one patient with ALS and one patient with FTD, respectively, ${ }^{819}$ as well as two missense, p.Asp118Asn and p.Ile397Thr, and one intronic variant, c.1644-5_1644-2delAATA, never associated with a clinical phenotype or ALS and reported at low frequency in the general population.

A number of TBK1 LoF pathogenic variants ${ }^{8}$ has been described so far, severely reducing the protein levels and suggesting a pathogenic mechanism based on haploinsufficiency. ${ }^{19}{ }^{21}$ In the present study, using functional in vitro assays we showed that the p.Leu59Phefs*16 frameshift results in undetectable TBK1 protein product, with consequent loss of both its catalytic function and binding ability. Also for the novel splice site c. $358+5 \mathrm{G}>\mathrm{A}$, we were able to demonstrate a loss of function since this variant resulted in exon 4 skipping, introduction of a PTC and ultimately to a $50 \%$ loss of transcript and protein levels in the patient, heterozygous for this variant. Interestingly, the previously described LoF variant, c. $358+2 \mathrm{~T}>\mathrm{C}$, located in the same donor splice site, was similarly not expressed at the protein level, but able to induce NMD through a different mechanism, namely intron 4 retention. ${ }^{8}$ The p.L59Ffs*16 and c. $358+5 \mathrm{G}>\mathrm{A}$ variants can be, therefore, considered as novel LoF pathogenic mutations, leading to TBK1 haploinsufficiency.

In addition to LoF mutations (null alleles), TBK1 functional impairment may also result from missense variants. Indeed, mutations in the $\mathrm{KD}$ may affect the protein catalytic activity, whereas mutations in the CCD1 may impair association with binding partners, like OPTN, even though it is still not clear which functions of TBK1 are relevant for neurodegeneration. ${ }^{21}$ Here, we showed that the novel p.Asp118Asn variant, located in the $\mathrm{KD}$, exhibited reduced levels of phosphorylation activity without affecting unchanged protein levels, consistently with our structure-based analysis results and supporting its role as a potentially pathogenic functional missense mutation. This conclusion was also supported by the observed high evolutionary conservation of the Asp118 codon, up to zebrafish. Similarly, we confirmed that previous findings for the p.Arg357Gln missense variant, located in the ULD, were able to impair TBK1 catalytic activity. ${ }^{8}$ Intriguingly, this reduction seemed related to a decrease of TBK1 protein levels rather than an intrinsic catalytic defect. Indeed, impairment of the catalytic activity might also be due to a combination of both loss-of-protein and protein function, which might in turn be due to instability of the mutated protein, a mechanism previously hypothesised for the p.L94S missense mutation. ${ }^{20}$ In contrast, the p.Ile397Thr variant exhibited a substantial increase in TBK1 phosphorylation activity, partly associated with higher protein levels. We speculate that the p.Ile397Thr variant may trigger an alternative dominant pathogenic mechanism involving enhanced TBK1 kinase activity and/or reduced protein turnover that might be as disruptive for cellular metabolism as the decrease caused by other pathogenic TBK1 mutations. However, the potential biological relevance of an increase of TBK1 phosphorylation activity or an imbalance of TBK1 protein turnover is not clear with respect to ALS/FTD pathogenesis and requires further investigation.

Our data support the wide heterogeneity of TBK1 related phenotypes, consistently with previous findings. ${ }^{20}{ }^{22-24}$ Age of onset in our mutated patients ranged from 36 to 81 years. Therefore, TBK1 mutations may also be considered in earlyonset patients with ALS. Moreover, while all our patients had 
A
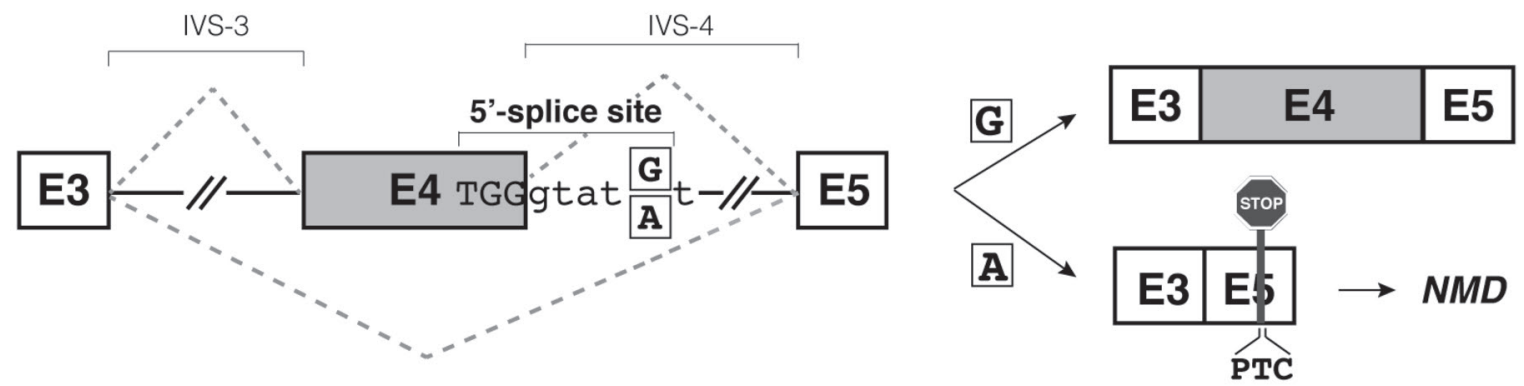

B

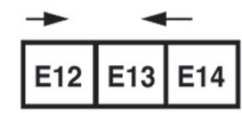

CNTR

C. $358+5 \mathrm{~A}$
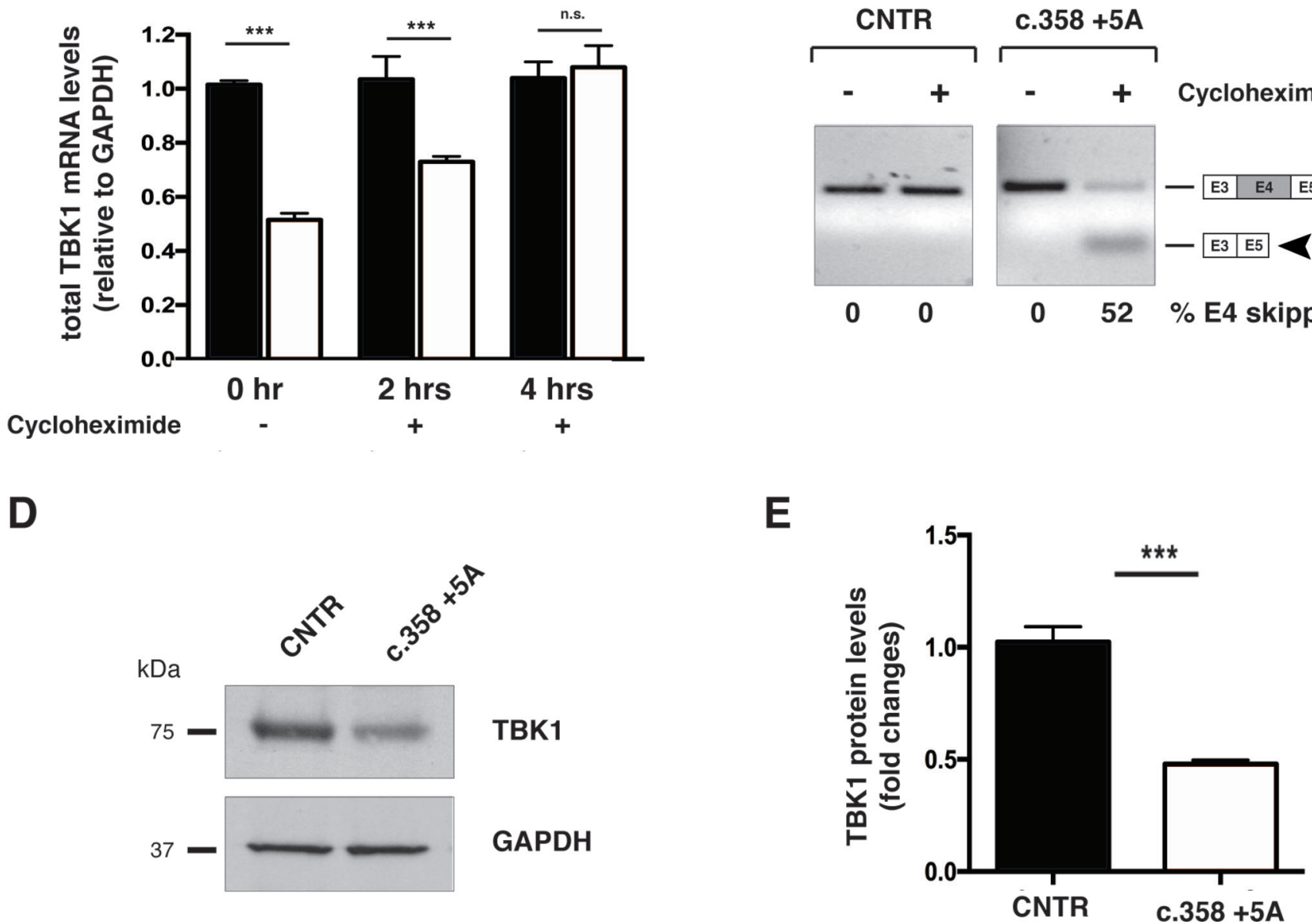

Figure 1 c.358+5A variant causes aberrant splicing of exon 4 leading to TBK1 haploinsufficiency. (A) Schematic representation of wild-type (c.358+5G) and mutated (c.358+5A) sequences. Exons are depicted as boxes (E3, E4 and E5) and introns (interVening sequence (IVS-3 and IVS-4) as lines. The sequence surrounding exon 4 donor splice site of the wild-type $G$ and mutant $A$ is indicated (position +5 ). The two possible splicing patterns (E4 inclusion or skipping) are indicated with diagonal dashed lines and the two possible resulting splicing products schematically reported on the right of the panel. (B) Analysis of total TBK1 mRNA levels by quantitative RT-PCR. Total RNA was extracted from primary control and c.358+5G/A fibroblasts at different time points (0, 2 and 4 hours) after cycloheximide administration. Arrows correspond to TBK1_E12/13 Fw and TBK1_E13 Rev quantitative PCR; primers. Results are expressed as mean \pm SD of three different experiments performed in triplicate. (C) TBK1 exon 4 splicing assay. Total RNA from samples in (B) was analysed by semiquantitative RT-PCR using TBK1_E2/3 Fw and TBK1_E5 Rev to detect inclusion or skipping (arrowhead) of exon 4 (see schematic diagrams in A). Band intensities were quantified and the percentage of exon 4 skipping is reported under each lane of the representative gel. (D) Immunoblot analysis of TBK1 protein levels in control and c.358+5G/A patient primary fibroblasts. GAPDH serves as a loading control. (E) Quantification of band intensity in (D) shows fold change reduction in c.358+5G/A fibroblasts relative to controls (mean \pm SD from three independent experiments). ${ }^{* * *} \mathrm{p}$-value $<0.001$. GAPDH, glyceraldehyde 3-phosphate dehydrogenase; NMD, non-sense mediated decay; n.S., not significant (Student's t-test); RT-PCR, real-time PCR; TBK1, TANKbinding kinase 1, CNTR: Control. 
A

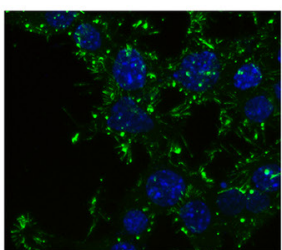

TBK1 WT

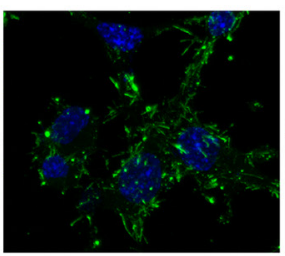

p.R357Q

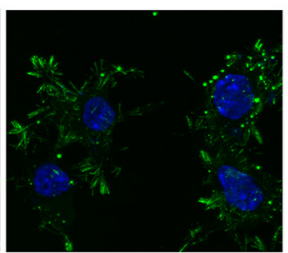

p.D118N

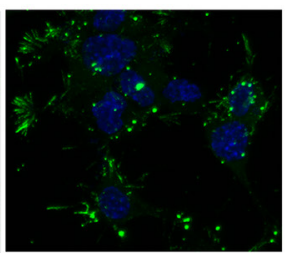

p.I397T

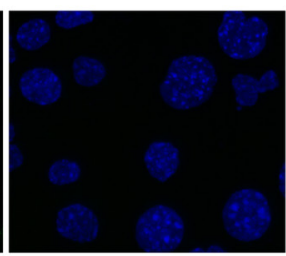

p.L59Ffs*
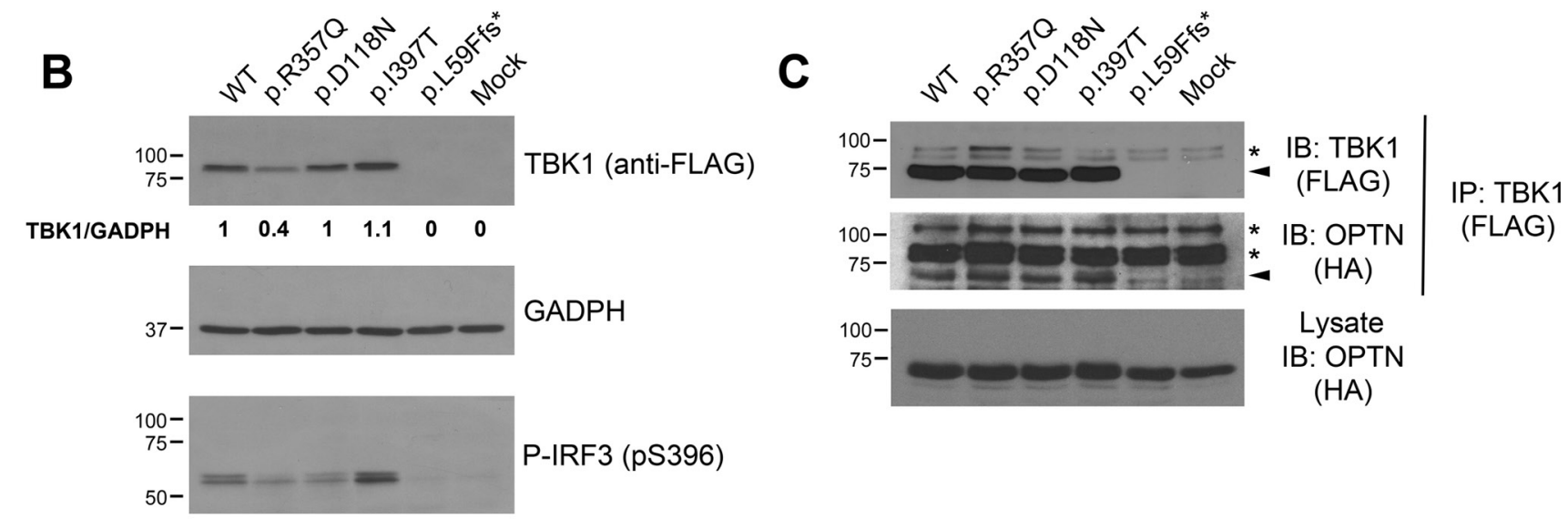

Lysate

IB: OPTN

(HA)

Figure 2 In vitro functional characterisation of TBK1 missense variants. (A) Subcellular distribution of TBK1 mutants visualised by anti-FLAG staining in differentiated motor neuron-like NSC-34 cells expressing the indicated tagged constructs. Similarly to wild-type TBK1, mutants localise in the cytosol, vesicular structures and lamellar domains at the cell periphery. L59Ffs $16-$ TBK1 is undetected. (B) Expression levels and kinase activity of TBK1 mutants (Top panels). Western blot analysis of FLAG-tagged wild-type TBK1 and indicated variants expressed in human embryonic kidney (AD293) cells along with HA-tagged IRF3. Green fluorescent protein (GFP) transfected cells serve as control (mock). Quantification of steady-state protein levels of TBK1 mutants relative to wild-type and normalised to GADPH is reported below each lane. The steady-state protein levels are lower for the p.Arg357Gln and slightly higher for the p.lle397Thr mutants. The truncating mutant p.Leu59Phefs* 16 abolishes TBK1 expression. Phosphorylation of IRF3 on Ser-396 in the same protein lysates was revealed with a phospho-specific antibody (bottom panels). The ratio between p-IRF3 and total IRF3 is reported under the lanes ( $p$-IRF3/tot IRF3) and these values are further normalised to the expression levels of each TBK1 mutant protein (p-IRF3/TBK1). Phosphorylation of IRF3 is impaired by the p.Asp118Asn mutation and enhanced by the p.lle397Thr. (C) TBK1 mutants interact with OPTN. FLAG-tagged constructs of wild-type TBK1 and indicated missense variants (or GFP control plasmid; 'mock') were expressed in AD293 cells together with HA-tagged OPTN. TBK1 constructs were pull-down with anti-FLAG antibody; OPTN in the immunocomplexes was revealed with anti-HA antibody. Membranes were stripped and reprobed with anti-FLAG antibody to detect the immunoprecipitated TBK1 constructs. Arrows mark target proteins, whereas asterisks point to non-specific bands. (In the figure aminoacids are indicated with the one-letter code instead of the three-letter code). GAPDH, glyceraldehyde 3-phosphate dehydrogenase; HA, haemagglutinin; OPTN, optineurin; p-IRF3, phosphorylated IRF3; TBK1, TANK-binding kinase 1; WT, wild type; IB: Immunoblot.

spinal onset, a wide spectrum of clinical phenotype could be observed, ranging from classic ALS to flail arm and pure lower motor neuron ALS. Cognitive impairment was observed in three patients, ${ }^{25}$ supporting the role of TBK1 mutations in the ALSFTD spectrum and further emphasising the concept that ALS and FTD share a common molecular and genetic background. ${ }^{8} 19$

Overall, in our cohort of Italian patients with ALS the observed frequency of TBK1 LoF mutations was 1.3\% (2/154), increasing up to $3.2 \%(5 / 154)$ by taking into account also the functional missense variants that we were able to classify as potentially pathogenic. This frequency is comparable with respect to previous ALS cohorts 8192026 and slightly higher in comparison to that reported in a Sardinian isolated population $(1.6 \%) .^{22}$ However, we acknowledge as a limitation that this is not a population-based study; therefore, the genetic epidemiology of TBK1 variants in the Italian ALS population requires further confirmation. Interestingly, in our cohort, LoH mutations were detectedin two 36-year-old woman, suggesting a potential gender effect. However, this hypothesis is not supported by a review of the literature of all previously reported cases, showing a balanced male to female ratio and no relevant gender effect on age of onset. TBK1 pathogenic variants were found in apparently patients with sALS, whereas no variants were detected in our fALS population; however, two patients had a positive family history for unclassified dementia suggesting a potential intrafamiliar overlap within the FTLD (frontotemporal lobar degeneration) spectruNotably, our patients harbouring the already reported p.Arg $357 \mathrm{Gln}^{8}$ and p.Lys $291 \mathrm{Glu}^{19}{ }^{20}$ functional missense variants presented with a phenotype differing from previously described patients with regard to age, site of onset 
and disease duration. Furthermore, the p.Lys291Glu, located in the KD and able to impair TBK1 phosphorylating activity, has been previously reported in one patient with FTD and also in two control subjects. ${ }^{19}{ }^{20}$ Altogether, these findings support the hypothesis that TBK1 pathogenic variants have a reduced penetrance and may be associated with a considerable phenotypic heterogeneity within the ALS/FTD spectrum, as also observed in multigenerational pedigrees segregating a TBK1 mutation, ${ }^{819} 2027$ and similarly to what is described for C9orf72 repeat expansions. ${ }^{28}$ However, a contribution of de novo mutations, compound heterozygous variants or genetic variants in multiple genes, consistent with an oligogenic model of disease may be taken into consideration. ${ }^{129}$

In conclusion, although the specific pathomechanism driven by TBK1 is still poorly understood, functional studies can provide critical evidence for the pathogenicity of unreported TBK1 variants, especially when segregation studies are not feasible. This study supports the relevance of TBK1 gene mutations in the Italian ALS and ALS-FTD spectrum population, suggesting its inclusion in the panel of genes to be sequenced in ALS.

\section{Author affiliations}

${ }^{1}$ Neuropathology Unit, Institute of Experimental Neurology (INSPE), Division of Neuroscience, San Raffaele Scientific Institute, Milan, Italy

${ }^{2}$ Molecular Neurobiology Laboratory, Division of Neuroscience, San Raffaele Scientific Institute, Milan, Italy

${ }^{3}$ Medical Genetic Unit, Department of Laboratory Medicine, Niguarda Hospital, Milan, Italy

${ }^{4}$ Department of Neurology, Institute of Experimental Neurology (INSPE), Division of Neuroscience, San Raffaele Scientific Institute, Milan, Italy

${ }^{5}$ Neurology Unit, "San Gerardo" Hospital and University of Milano-Bicocca, Monza, Italy

${ }^{6}$ Department of Neurosciences, Neuromuscular Center, University of Padova, Padua, Italy

${ }^{7}$ Neuroimaging Research Unit, Department of Neurology, Institute of Experimental Neurology (INSPE), Division of Neuroscience, San Raffaele Scientific Institute, Milan, Italy

${ }^{8}$ Università Vita e Salute San Raffaele, Milan, Italy

${ }^{9}$ Division of Genetics and Cell Biology, Unit of Genomics for Human Disease Diagnosis, San Raffaele Scientific Institute, Milan, Italy

${ }^{10}$ NEuroMuscular Omnicentre (NEMO), Fondazione Serena Onlus, Milan, Italy

Acknowledgements The authors thank Linda Ottoboni and Francesca Ruffini for technical assistance.

Contributors $L P, F V, L M, A D M, T D, A R$ and $C T$ performed the experiments and acquired the data; LP, FV, LM, ADM, TD, AR, FA, SP, DB, PC and NR contributed to analysis and interpretation of the data; YMF, LT, GS, FC, RF, FA, CL and NR recruited the patients, performed the clinical evaluation, acquired clinical data and collected patient DNA; LP, FV, ADM, AR, YMF, DB, PC and NR drafted and revised the manuscript; $L T, G S, F A, G C, M F, A Q, C L, S P, D B, P C$ and NR critically revised the manuscript for intellectual content; $A Q, C L, S P, D B, P C$ and NR conceptualised and designed the study, and interpreted the analysis; FA, GC, MF, AQ, CL, SP, DB, PC and NR obtained the funding; $A Q, S P, D B, P C$ and NR supervised the study; all authors had full access to the data in the study and approved the final version of the manuscript.

Funding This work was supported by AriSLA, Italian Research Foundation for ALS (ExAlta Grant) to AQ, the Giovanni Armenise-Harvard Foundation and ERC-StG 35590-NEVAI to DB and the Italian Ministry of Health (grant RF-2010-2313220) to AF.

Competing interests None declared.

Patient consent Obtained

Ethics approval The study was approved by the local Ethical Committee of the San Raffaele Scientific Institute.

Provenance and peer review Not commissioned; externally peer reviewed.

Open Access This is an Open Access article distributed in accordance with the Creative Commons Attribution Non Commercial (CC BY-NC 4.0) license, which permits others to distribute, remix, adapt, build upon this work non-commercially, and license their derivative works on different terms, provided the original work is properly cited and the use is non-commercial. See: http://creativecommons.org/ licenses/by-nc/4.0/

(c) Article author(s) (or their employer(s) unless otherwise stated in the text of the article) 2017. All rights reserved. No commercial use is permitted unless otherwise expressly granted.

\section{REFERENCES}

1 Riva N, Agosta F, Lunetta C, et al. Recent advances in amyotrophic lateral sclerosis. J Neurol 2016;263:1241-54.

2 Lomen-Hoerth C, Anderson T, Miller B. The overlap of amyotrophic lateral sclerosis and frontotemporal dementia. Neurology 2002;59:1077-9.

3 Goldstein LH, Abrahams S. Changes in cognition and behaviour in amyotrophic lateral sclerosis: nature of impairment and implications for assessment. Lancet Neurol 2013;12:368-80.

4 Renton AE, Chiò A, Traynor BJ. State of play in amyotrophic lateral sclerosis genetics. Nat Neurosci 2014;17:17-23.

5 Hardiman 0, Al-Chalabi A, Brayne C, et al. The changing picture of amyotrophic lateral sclerosis: lessons from European registers. I Neurol Neurosurg Psychiatry 2017;88:557-63.

6 Veldink JH. ALS genetic epidemiology 'How simplex is the genetic epidemiology of ALS?'. J Neurol Neurosurg Psychiatry 2017:88:537.

7 Cirulli ET, Lasseigne BN, Petrovski S, et al. Exome sequencing in amyotrophic lateral sclerosis identifies risk genes and pathways. Science 2015;347:1436-41.

8 Freischmidt A, Wieland T, Richter B, et al. Haploinsufficiency of TBK1 causes familial ALS and fronto-temporal dementia. Nat Neurosci 2015;18:631-6.

9 Pottier C, Bieniek KF, Finch N, et al. Whole-genome sequencing reveals important role for TBK 1 and OPTN mutations in frontotemporal lobar degeneration without motor neuron disease. Acta Neuropathol 2015;130:77-92.

10 Ahmad L, Zhang SY, Casanova JL, et al. Human TBK1: a gatekeeper of neuroinflammation. Trends Mol Med 2016:22:511-27.

11 Oakes JA, Davies MC, Collins MO. TBK1: a new player in ALS linking autophagy and neuroinflammation. Mol Brain 2017:10:5.

12 Wild P, Farhan H, McEwan DG, et al. Phosphorylation of the autophagy receptor optineurin restricts Salmonella growth. Science 2011;333:228-33.

13 Pilli M, Arko-Mensah J, Ponpuak M, et al. TBK-1 promotes autophagy-mediated antimicrobial defense by controlling autophagosome maturation. Immunity 2012:37:223-34.

14 Brooks BR, Miller RG, Swash M, et al. El Escorial revisited: revised criteria for the diagnosis of amyotrophic lateral sclerosis. Amyotroph Lateral Scler Other Motor Neuron Disord 2000;1:293-9.

15 Byrne S, Bede P, Elamin M, et al. Proposed criteria for familial amyotrophic lateral sclerosis. Amyotroph Lateral Scler 2011;12:157-9.

16 Chiò A, Calvo A, Moglia C, et al. Phenotypic heterogeneity of amyotrophic lateral sclerosis: a population based study. J Neurol Neurosurg Psychiatry 2011;82:740-6.

17 Baker NA, Sept D, Joseph S, et al. Electrostatics of nanosystems: application to microtubules and the ribosome. Proc Natl Acad Sci U S A 2001;98:10037-41.

18 Guerois R, Nielsen JE, Serrano L. Predicting changes in the stability of proteins and protein complexes: a study of more than 1000 mutations. J Mol Biol 2002;320:369-87.

19 Giijselinck I, Van Mossevelde S, van der Zee J, et al. Loss of TBK1 is a frequent cause of frontotemporal dementia in a Belgian cohort. Neurology 2015;85:2116-25.

20 van der Zee J, Gijselinck I, Van Mossevelde S, et al. TBK1 Mutation Spectrum in an Extended European Patient Cohort with Frontotemporal Dementia and Amyotrophic Lateral Sclerosis. Hum Mutat 2017;38:297-309.

21 Freischmidt A, Müller K, Ludolph AC, et al. Association of mutations in TBK1 with sporadic and familial amyotrophic lateral sclerosis and frontotemporal dementia. JAMA Neurol 2017:74:110-3.

22 Borghero G, Pugliatti M, Marrosu F, et al. TBK1 is associated with ALS and ALS-FTD in sardinian patients. Neurobiol Aging 2016:43:180.e1-180.e5.

23 Tsai PC, Liu YC, Lin KP, et al. Mutational analysis of TBK1 in Taiwanese patients with amyotrophic lateral sclerosis. Neurobiol Aging 2016:40:191.e11-191.e16.

24 Gomez-Tortosa E, Van der Zee J, Ruggiero Mara, et al. Familial primary lateral sclerosis or dementia associated with Arg573Gly TBK1 mutation J Neurol Neurosurg Psychiatry 2017. doi: 10.1136/jnnp-2016-315250. [Epub ahead of print].

25 Montuschi A, lazzolino B, Calvo A, et al. Cognitive correlates in amyotrophic lateral sclerosis: a population-based study in Italy. J Neurol Neurosurg Psychiatry 2015;86:168-73.

26 Le Ber I, De Septenville A, Millecamps S, et al. TBK1 mutation frequencies in French frontotemporal dementia and amyotrophic lateral sclerosis cohorts. Neurobiol Aging 2015:36:3116.e5-3116.e8.

27 Van Mossevelde S, van der Zee J, Gijselinck I, et al. Clinical features of TBK1 carriers compared with C9orf72, GRN and non-mutation carriers in a Belgian cohort. Brain 2016;139(Pt 2):452-67.

28 Hübers A, Marroquin N, Schmoll B, et al. Polymerase chain reaction and Southern blot-based analysis of the C9orf72 hexanucleotide repeat in different motor neuron diseases. Neurobiol Aging 2014;35:1214.e1-1214.e6.

29 van Blitterswijk M, Hennekam EA, et al. Evidence for an oligogenic basis of amyotrophic lateral sclerosis. Hum Mol Genet 2012;21:3776-84. 